\title{
The prognostic relevance of p53 and Ki-67 to chemotherapy sensitivity and prognosis in triple-negative breast cancer
}

\author{
Guojing Zhang ${ }^{1}$, Zhongyi Shi ${ }^{2}$, Lina Liu ${ }^{3}$, Heqing Yuan ${ }^{1}$, Zheng Pan $^{1}$, Wenxu Li $^{1}$, Yu Tao ${ }^{1}$, \\ Zhaoming Huang', Xiaoying Huang ${ }^{2}$, Chao Lin ${ }^{3}$ \\ ${ }^{1}$ Oncology Department, Wenzhou Geriatric Hospital, Wenzhou, China; ${ }^{2}$ Tumor Surgery Department, Wenzhou Hospital of Traditional Chinese \\ Medicine, Wenzhou, China; ${ }^{3}$ Gynecology Department, Wenzhou Hospital of Traditional Chinese Medicine, Wenzhou, China \\ Contributions: (I) Conception and design: G Zhang, Z Shi, C Lin; (II) Administrative support: Z Shi; (III) Provision of study materials or patients: L \\ Liu, C Lin; (IV) Collection and assembly of data: H Yuan, Z Pan, W Li, Y Tao; (V) Data analysis and interpretation: H Yuan, Z Pan; (VI) Manuscript \\ writing: All authors; (VII) Final approval of manuscript: All authors. \\ Correspondence to: Chao Lin. Gynecology Department, Wenzhou Hospital of Traditional Chinese Medicine. No.9 Jiaowei Road, Liuhongqiao, \\ Wenzhou 325000, China. Email: linchao59@163.com.
}

Background: This study aimed to explore the prognostic function of p53 and Ki-67 protein expression in chemotherapy sensitivity and prognosis in triple-negative breast cancer (TNBC).

Methods: Patients who were confirmed with TNBC in Wenzhou Geriatric Hospital and Wenzhou Hospital of Traditional Chinese Medicine (including the Oncology Department, Tumor Surgery Department, and Gynecology Department) between January 2006 and February 2018 were included in this study. The expression of p53 and Ki-67 detected by immunohistochemistry, the rate of recurrence, and the objective curative effect evaluation at the end of the first-line rescue treatment were recorded for all patients. Results: The patients were followed up to August 2020, and the median follow-up time was 9 years and 4 months. A total of 285 patients with TNBC were enrolled in the study. The patients ranged in age from 19 to 76 years old, with an average age of 53 years. The overall recurrence rate among the patients was $31.58 \%$. The majority of cases $(68.07 \%)$ were pathological stage I. The overall positive expression rates of $\mathrm{Ki}-67$ and p53 were $53.33 \%$ and $56.84 \%$, respectively. In the TNBC recurrence group, the positive rates of p53 and $\mathrm{Ki}-67$ were $71.11 \%$ and $82.22 \%$, respectively, which were significantly higher than those in the non-recurrence group. The positive rates of p53 and Ki-67 in the chemosensitive group were $96.05 \%$ and $92.11 \%$, respectively, which were significantly higher than those in the non-chemosensitive group. Among all the TNBC patients, 128 patients had positive expression of both $\mathrm{p} 53$ and $\mathrm{Ki}-67$, and 101 patients had negative expression of both $\mathrm{p} 53$ and $\mathrm{Ki}-67$. The chemosensitivity rate of TNBC patients with positive expression of both $\mathrm{Ki}-67$ and p53 was $98.53 \%$, and that of TNBC patients with negative expression of both Ki-67 and p53 was $0.00 \%$. The difference was statistically significant. The recurrence rate in TNBC patients with positive expression of both $\mathrm{Ki}-67$ and p53 was $53.13 \%$, and that in patients with negative expression of both $\mathrm{Ki}-67$ and p53 was $6.93 \%$. The difference was statistically significant.

Conclusions: The expression of $\mathrm{p} 53$ and $\mathrm{Ki}-67$ had prognostic relevance to chemotherapy sensitivity and prognosis in TNBC patients.

Keywords: Triple-negative breast cancer (TNBC); Ki-67; p53; chemotherapy sensitivity; risk of recurrence

Submitted Jan 11, 2021. Accepted for publication Feb 20, 2021.

doi: $10.21037 /$ tcr-21-180

View this article at: http://dx.doi.org/10.21037/tcr-21-180 


\section{Introduction}

Triple-negative breast cancer (TNBC), which was first described by Brenton et al. in 2005 (1), refers to breast cancer that is negative for hormone receptors and human epidermal growth factor receptor 2 (HER2). TNBC is a complex subtype that represents approximately $12 \%$ to $17 \%$ of all breast cancers (2), and most frequently occurs in premenopausal and young ( $<40$ years old) women (3). TNBC displays especial biological behavior, pathological characteristics, and clinical manifestations. TNBC is not sensitive to endocrine therapy or targeted therapy, and is highly malignant with a high rate of distant metastasis.

Chemotherapy is the only systemic treatment available for TNBC at present (4). The expression of $\mathrm{p} 53$ is a potential predictor of sensitivity to chemotherapy in breast cancer, especially for patients with luminal breast cancer, but its prognostic function in TNBC is poor (5). Lee et al. reported that the chemotherapeutic effect on TNBCs with high p53 expression was greater than that on TNBCs with low p53 expression (6), but a later study found the opposite (7). $\mathrm{Ki}-67$ is one of the most widely used tumor proliferationrelated biomarkers, and it can predict the chemosensitivity of TNBC (8). Although chemotherapy has been shown to be effective in the treatment of TNBC under the guidance of some predictive markers, it has a high recurrence rate and short overall survival (9).

Recurrence is an independent prognostic factor for survival of TNBC; thus, illuminating the relationship between tumor markers and recurrence would assist in improving the prognosis of TNBC. In this study, we aimed to investigate the prognostic function of $\mathrm{p} 53$ and $\mathrm{Ki}-67$ protein expression in chemosensitivity and prognosis in patients with TNBC.

We present the following article in accordance with the REMARK reporting checklist (available at http://dx.doi. org/10.21037/tcr-21-180).

\section{Methods}

\section{General information}

Patients who were confirmed with TNBC by pathological examination between January 2006 and February 2018 in Wenzhou Geriatric Hospital and Wenzhou Hospital of Traditional Chinese Medicine (including the Oncology Department, Tumor Surgery Department, and Gynecology Department) were enrolled in this study. The patients were followed up to August, 2020, and the median follow-up time was 9 years and 4 months.

All procedures performed in this study involving human participants were in accordance with the Declaration of Helsinki (as revised in 2013). The study was approved by ethics board of Wenzhou Geriatric Hospital (NO.: WLNY2020-005). Because of the retrospective nature of the research, the requirement for informed consent was waived.

\section{Inclusion criteria}

Immunohistochemical (IHC) detection of estrogen receptor (ER), progesterone receptor (PR), HER2, Ki-67, and p53 was performed as standard, using the following definitions: ER positivity: $\geq 1 \%$ ER-positive cells; PR positivity: $\geq 1 \%$ PRpositive cells; HER 2 positivity: $10 \%$ of tumor cell membranes uniform and strong staining; 553 positivity: positive p53 protein expression with brown-yellow nuclear staining, and $>10 \%$ tissue cells stained positive; and Ki-67 positivity: positive Ki-67 protein expression with brown-yellow nuclear staining, and $>14 \%$ tissue cells stained positive.

Only breast cancers confirmed as being triple negative for ER, PR, and HER2 were included in the study. All patients were female, and none of the patients had distant metastasis or other primary malignant tumors. All patients received surgical treatment and had a pathological diagnosis. None of the patients received radiotherapy or chemotherapy preoperatively. All patients received standardized adjuvant treatment postoperatively and had complete clinical followup data.

\section{Data}

All patients were classified according to age, tumor size, menopausal status, lymph node status, tumor-node-metastasis (TNM) stage, histological grading, p53 and Ki-67 expression, whether or not relapse occurred, chemosensitivity.

\section{Criteria for treatment sensitivity}

After multiple courses of treatment, tumor cells may undergo heterogeneous changes and subsequently develop insensitivity to chemotherapy. Therefore, we selected the effectiveness of first-line rescue chemotherapy as the reference for treatment sensitivity. After the completion of first-line chemotherapy, the objective curative effect was evaluated according to the American Cancer Society's Response Evaluation Criteria in Solid Tumors (RECIST) version 1.1 as follows: complete remission (CR): the disappearance of all target lesions; partial 
Table 1 Clinicopathological characteristics of 285 TNBC patients

\begin{tabular}{|c|c|c|}
\hline Clinicopathological characteristics & No. of cases & $\%$ \\
\hline \multicolumn{3}{|l|}{ Age (years) } \\
\hline$<50$ & 161 & 56.49 \\
\hline$\geq 50$ & 124 & 43.51 \\
\hline \multicolumn{3}{|l|}{ Menopausal status } \\
\hline Premenopausal & 166 & 58.25 \\
\hline Postmenopausal & 119 & 41.75 \\
\hline \multicolumn{3}{|l|}{ Tumor size (pT) } \\
\hline$\leq 2 \mathrm{~cm}$ & 143 & 50.18 \\
\hline$>2 \mathrm{~cm}$ & 142 & 49.82 \\
\hline \multicolumn{3}{|l|}{ Lymph node status (pN) } \\
\hline Yes & 141 & 49.47 \\
\hline No & 144 & 50.53 \\
\hline \multicolumn{3}{|l|}{ Pathological stage (pTNM) } \\
\hline I & 194 & 68.07 \\
\hline II-III & 91 & 31.93 \\
\hline \multicolumn{3}{|l|}{ Histological grading } \\
\hline I-II & 204 & 71.58 \\
\hline III & 81 & 28.42 \\
\hline \multicolumn{3}{|l|}{ p53 } \\
\hline Negative & 123 & 43.16 \\
\hline Positive & 162 & 56.84 \\
\hline \multicolumn{3}{|l|}{$\mathrm{Ki}-67$} \\
\hline Negative & 133 & 46.67 \\
\hline Positive & 152 & 53.33 \\
\hline \multicolumn{3}{|l|}{ Relapse } \\
\hline Yes & 90 & 31.58 \\
\hline No & 195 & 68.42 \\
\hline
\end{tabular}

TNBC, triple-negative breast cancer; pT, pathology tumor; pN, pathology node; pTNM, pathology tumor node metastasis.

remission (PR): total diameter of target lesions reduced by $\geq 30 \%$ from baseline; progressive disease (PD): total diameter of target lesions increased by $\geq 20 \%$ from baseline or the appearance of new lesions; stable disease (SD): a decrease or increase in the total long diameters of the target lesions from baseline without reaching PR or PD. Tumors evaluated as CR or PR were regarded as being sensitive to chemotherapy, while those evaluated as SD or PD were regarded as being insensitive.

\section{Data processing}

Data on Ki-67 and p53 expression, chemosensitivity, and recurrence were extracted for all cases. Microsoft Office Excel software was used to establish a database. SPSS 20.0 software was used to process the data statistically. The number of cases in each group was tested for normal distribution.

\section{Results}

\section{General clinical data}

A total of 285 patients with TNBC were enrolled in the study. The patients ranged in age between 19 and 76 years old, with a median age of 53 years old. Pathologic stage I accounted for $68.07 \%$ of cases. The positive expression rates of $\mathrm{Ki}-67$ and p 53 were $53.33 \%$ and $56.84 \%$, respectively. The overall recurrence rate was $31.58 \%$. The clinical pathological characteristics of all the TNBC patients are shown in Table 1.

\section{The prognostic function of Ki-67 in chemosensitivity and recurrence in TNBC}

The positive expression rate of $\mathrm{Ki}-67$ in chemosensitive and non-chemosensitive cases was $92.11 \%$ and $21.43 \%$, respectively, and the difference was statistically significant. Among the patients who experienced relapse, the positive expression rate of $\mathrm{Ki}-67$ was $82.22 \%$, compared with a rate of $40.00 \%$ in patients without recurrence, constituting a statistically significant difference (Table 2).

\section{The prognostic function of $p 53$ in chemosensitivity and recurrence in TNBC}

The positive expression rate of p53 was $96.05 \%$ in chemosensitive patients and $35.71 \%$ in non-chemosensitive patients, and the difference was statistically significant. In patients who suffered recurrence, the positive expression rate of p53 was $71.11 \%$, which was statistically significantly higher than the rate of $56.41 \%$ observed in patients without recurrence (Table 3).

\section{The prognostic function of simultaneous expression of Ki-} 67 and p53 in chemosensitivity and recurrence in TNBC

The rate of chemosensitivity in TNBC patients with positive expression of both $\mathrm{Ki}-67$ and $\mathrm{p} 53$ protein reached $98.53 \%$, while the chemosensitivity rate of TNBC patients 
Table 2 The prognostic function of Ki-67 in chemosensitivity and recurrence in triple-negative breast cancer (TNBC)

\begin{tabular}{|c|c|c|c|c|}
\hline & \multicolumn{2}{|c|}{ Ki-67 } & $\chi^{2}$ & $\mathrm{P}$ \\
\hline Chemosensitivity & & & 38.545 & $<0.001$ \\
\hline Sensitive & $6(7.89 \%)$ & 70 (92.11\%) & & \\
\hline Not sensitive & $11(78.57 \%)$ & $3(21.43 \%)$ & & \\
\hline Relapse & $16(17.78 \%)$ & $74(82.22 \%)$ & & \\
\hline No recurrence & 117 (60.00\%) & $78(40.00 \%)$ & & \\
\hline
\end{tabular}

Table 3 The prognostic function of $\mathrm{p} 53$ in chemosensitivity and recurrence in triple-negative breast cancer (TNBC)

\begin{tabular}{|c|c|c|c|c|}
\hline & \multicolumn{2}{|c|}{ P53 } & $\chi^{2}$ & $\mathrm{P}$ \\
\hline Chemosensitivity & & & 37.247 & $<0.001$ \\
\hline Sensitive & 3 (3.95\%) & 73 (96.05\%) & & \\
\hline Not sensitive & 9 (64.29\%) & $5(35.71 \%)$ & & \\
\hline Relapse & 12 (28.89\%) & 78 (71.11\%) & & \\
\hline No recurrence & $111(43.59 \%)$ & $84(56.41 \%)$ & & \\
\hline
\end{tabular}

Table 4 The prognostic function of simultaneous expression of Ki-67 and p53 in triple-negative breast cancer (TNBC) chemosensitivity

\begin{tabular}{lccc}
\hline \multirow{2}{*}{ Ki-67 and P53 } & \multicolumn{2}{c}{ Chemosensitivity } & $\chi^{2}$ \\
\cline { 2 - 3 } & Sensitive & Not sensitive & P \\
\hline Negative & $0(0.00 \%)$ & $7(100.00 \%)$ & 64.66 \\
Positive & $67(98.53 \%)$ & $1(1.47 \%)$ & $<0.001$ \\
\hline
\end{tabular}

with negative expression of both $\mathrm{Ki}-67$ and $\mathrm{p} 53$ protein was $0.00 \%$ (Table 4), showing a statistically significant difference. Among TNBC patients with positive expression of both $\mathrm{Ki}-67$ and p53 protein, the recurrence rate was $53.13 \%$, which was statistically significantly higher than the recurrence rate in patients with negative expression of both Ki-67 and p53 protein (6.93\%) (Table 5).

\section{Correlation analysis between $\mathrm{p} 53$ and $\mathrm{Ki}-67$ protein expression in $T \mathrm{NBC}$}

Among the TNBC patients, 128 patients had positive expression of both p53 and Ki-67, while 101 patients had negative expression of both p53 and Ki-67(Table 6). There was a positive correlation between the expression of p53 and $\mathrm{Ki}-67$ in $\mathrm{TNBC}(\mathrm{P}=0.000)$.

\section{Discussion}

TNBC is a systemic disease that can easily metastasize through the blood tract to the central nervous system and lungs during the early stage (10). Although chemotherapy is a highly effective treatment for TNBC, it is associated with a high rate of recurrence, which is attributable to the special biological behavior of this malignancy. Patients with TNBC who have poor sensitivity to chemotherapy 
Table 5 The prognostic function of simultaneous expression of Ki-67 and p53 in triple-negative breast cancer (TNBC) recurrence

\begin{tabular}{lclc}
\hline \multirow{2}{*}{ Ki-67 and P53 } & \multicolumn{2}{c}{ Recurrence } & $\chi^{2}$ \\
\cline { 2 - 4 } & Relapse & No recurrence & 54.697 \\
Negative & $7(6.93 \%)$ & $94(93.07 \%)$ & $<0.001$ \\
Positive & $68(53.13 \%)$ & $60(46.88 \%)$ & \\
\hline
\end{tabular}

Table 6 Spearman's correlation analysis between the expression of p53 and Ki-67 in triple-negative breast cancer (TNBC)

\begin{tabular}{lcccc}
\hline & Ki-67 (-) & Ki-67 (+) & $\chi^{2}$ & P \\
\hline P53 (-) & $101(35.44 \%)$ & $22(7.72 \%)$ & 104.784 & $<0.001$ \\
P53 (+) & $34(11.93 \%)$ & $128(44.91 \%)$ & \\
\hline
\end{tabular}

have unfavorable outcomes, with short overall survival, disease-free survival, and relapse-free survival (11). To further improve the treatment efficacy for TNBC patients, it is necessary to study the molecular mechanism of the occurrence and development of TNBC.

Ki-67 and p53 are 2 widely studied indicators, which serve as IHC markers in almost all hospitals. Investigation of the relationships between Ki-67, p53, chemosensitivity, and recurrence of TNBC can help to guide clinical practice. This study showed that Ki-67 and p53 were both independent indicators of chemosensitivity and recurrence in patients with TNBC. TNBC patients with high Ki-67 or $\mathrm{p} 53$ expression were more sensitive to chemotherapy and displayed a higher recurrence rate. Among the TNBC patients, 128 patients had positive expression of both p53 and Ki-67, and 101 patients had negative expression of both $\mathrm{p} 53$ and $\mathrm{Ki}-67$. We found a positive correlation between $\mathrm{p} 53$ and $\mathrm{Ki}-67$ protein expression in TNBC tissue, suggesting that $\mathrm{p} 53$ and $\mathrm{Ki}-67$ play synergistic roles in the growth of TNBC. The predictive function of Ki-67 and p53 was more obvious in TNBC patients with a positive expression of both markers.

In 2013, the definition of HER2-positive tumors in IHC was changed to include those with $10 \%$ uniformity of tumor cell membranes (reduced from 30\%) and strong staining (12). Some IHC reports show only positive or negative results, and do not describe the uniformity rate of tumor cell membranes or the strength of staining. In the present study, we eliminated cases that were not fully described by IHC, which might have led to a decrease in the number of enrolled cases.

Some previous studies have reported the recurrence rate of TNBC to be approximately $40 \%(13,14)$, which was slightly higher than the rate in this study. Zeng et al. found that a younger age at onset might indicate a higher degree of malignancy in TNBC (15), and the age of onset has also been identified as a factor related to recurrence (16). More than half of the cases in our study were under the age of 50 years old, which should have, on the basis of previous reports, led to an increase in the recurrence rate; however, our result was inconsistent with this. Further statistical analysis showed that 56 cases were operated on after 2015, and the follow-up time was less than 5 years. The shorter follow-up time might be the main reason for the low recurrence rate in our study. In the future, more accurate results will be obtained by extending the follow-up time and calculating the 2-, 5-, and 10-year recurrence rates.

\section{Acknowledgments}

Funding: This study was supported by Wenzhou Basic Medical and Health Science and Technology Project (no. Y20180836).

\section{Footnote}

Reporting Checklist: The authors have completed the REMARK reporting checklist. Available at http://dx.doi. org/10.21037/tcr-21-180

Data Sharing Statement: Available at http://dx.doi. org/10.21037/tcr-21-180

Conflicts of Interest: All authors have completed the ICMJE uniform disclosure form (available at http://dx.doi. org/10.21037/tcr-21-180). The authors have no conflicts of 
interest to declare.

Ethical Statement: The authors are accountable for all aspects of the work in ensuring that questions related to the accuracy or integrity of any part of the work are appropriately investigated and resolved. All procedures performed in this study involving human participants were in accordance with the Declaration of Helsinki (as revised in 2013). The study was approved by ethics board of Wenzhou Geriatric Hospital (NO.: WLNY2020-005). Because of the retrospective nature of the research, the requirement for informed consent was waived.

Open Access Statement: This is an Open Access article distributed in accordance with the Creative Commons Attribution-NonCommercial-NoDerivs 4.0 International License (CC BY-NC-ND 4.0), which permits the noncommercial replication and distribution of the article with the strict proviso that no changes or edits are made and the original work is properly cited (including links to both the formal publication through the relevant DOI and the license). See: https://creativecommons.org/licenses/by-nc-nd/4.0/.

\section{References}

1. Stathopoulos GP, Malamos NA, Markopoulos C, et al. The role of Ki-67 in the proliferation and prognosis of breast cancer molecular classification subtypes. Anticancer Drugs 2014;25:950-7.

2. Polonio-Alcalá E, Rabionet M, Gallardo X, et al. PLA Electrospun Scaffolds for Three-Dimensional TripleNegative Breast Cancer Cell Culture. Polymers (Basel) 2019;11:916.

3. Asano Y, Kashiwagi S, Onoda N, et al. Clinical verification of sensitivity to preoperative chemotherapy in cases of androgen receptor-expressing positive breast cancer. $\mathrm{Br} \mathrm{J}$ Cancer 2016;114:14-20.

4. Yagata H, Kajiura Y, Yamauchi H. Current strategy for triple-negative breast cancer: appropriate combination of surgery, radiation, and chemotherapy. Breast Cancer 2011;18:165-73.

5. Andre F, Pusztai L. Heterogeneity of breast cancer among patients and implications for patient selection for adjuvant chemotherapy. Pharm Res 2006;23:1951-8.

6. Bidard FC, Matthieu MC, Chollet P, et al. p53 status and efficacy of primary anthracyclines/alkylating agent-based regimen according to breast cancer molecular classes. Ann Oncol 2008;19:1261-5.
7. Lee HC, Ko H, Seol H, et al. Expression of

Immunohistochemical Markers before and after Neoadjuvant Chemotherapy in Breast Carcinoma, and Their Use as Predictors of Response. J Breast Cancer 2013;16:395-403.

8. Zhang G, Xie W, Liu Z, et al. Prognostic function of Ki67 for pathological complete response rate of neoadjuvant chemotherapy in triple-negative breast cancer. Tumori 2014;100:136-42.

9. Collins KAL, Stuhlmiller TJ, Zawistowski JS, et al. Proteomic analysis defines kinase taxonomies specific for subtypes of breast cancer. Oncotarget 2018;9:15480-97.

10. Shawky MS, Huo CW, Henderson MA, et al. A review of the influence of mammographic density on breast cancer clinical and pathological phenotype. Breast Cancer Res Treat 2019;177:251-76.

11. Kong X, Moran MS, Zhang N, et al. Meta-analysis confirms achieving pathological complete response after neoadjuvant chemotherapy predicts favourable prognosis for breast cancer patients. Eur J Cancer 2011;47:2084-90.

12. Turashvili G, Wen HY. Multigene testing in breast cancer: What have we learned from the 21-gene recurrence score assay? Breast J 2020;26:1199-207.

13. Carey LA, Rugo HS, Marcom PK, et al. TBCRC 001: randomized phase II study of cetuximab in combination with carboplatin in stage IV triple-negative breast cancer. J Clin Oncol 2012;30:2615-23.

14. Sikov WM, Berry DA, Perou CM, et al. Impact of the addition of carboplatin and/or bevacizumab to neoadjuvant once-per-week paclitaxel followed by dose-dense doxorubicin and cyclophosphamide on pathologic complete response rates in stage II to III triple-negative breast cancer: CALGB 40603 (Alliance). J Clin Oncol 2015;33:13-21.

15. Zeng Y, Wang CL, Xian J, et al. Positive correlation between programmed death ligand- 1 and $\mathrm{p} 53$ in triple-negative breast cancer. Onco Targets Ther 2019;12:7193-201.

16. Kurian AW, Ward KC, Howlader N, et al. Genetic Testing and Results in a Population-Based Cohort of Breast Cancer Patients and Ovarian Cancer Patients. J Clin Oncol 2019;37:1305-15.

(English Language Editor: J. Reynolds)

Cite this article as: Zhang G, Shi Z, Liu L, Yuan H, Pan Z, Li W, Tao Y, Huang Z, Huang X, Lin C. The prognostic relevance of p53 and $\mathrm{Ki}-67$ to chemotherapy sensitivity and prognosis in triple-negative breast cancer. Transl Cancer Res 2021;10(2):1082-1087. doi: 10.21037/tcr-21-180 